\title{
Modeling and optimization of vertical-external-cavity surface-emitting diode lasers for passive mode-locking
}

\author{
Josep Mulet and Salvador Balle \\ Institut Mediterrani d'Estudis Avançats (IMEDEA), CSIC-UIB, \\ Campus Universitat de les Illes Balears, E-07122 Palma de Mallorca, Spain
}

\begin{abstract}
We present a comprehensive description of electrically-driven vertical-external-cavity surface-emitting diode lasers (VECSELs) at $980 \mathrm{~nm}$, mode-locked by saturable absorber mirrors. A novel partially-integrated time-domain model combines accuracy and flexibility, allowing for a semi-analytical stability analysis of the compoundcavity modes, tracking the mode-locking onset and an optimization analysis. The linear stability analysis of the compound-cavity modes indicates that single mode solutions exist and are stable only in a limited current range around threshold. Increasing the current above this current level leads to a multimode solution through a Hopf bifurcation. This bifurcation point is followed by a continuous transition leading from harmonic oscillations to fully-developed pulses that correspond to the mode-locked solution. We obtain stable, fully-developed modelocked pulses of few tens of picoseconds at $15 \mathrm{GHz}$ repetition rate in good agreement with reported experimental results. We discuss the dependence of the mode-locking regimes on the reflectivity of the distributed Bragg reflectors, spot area of the spatial mode, and number of quantum wells in the emitter and absorber cavities. The optimization analysis reveals that, in order to favor the mode-locking onset, the effective coupling between the emitter and saturable absorber cavities has to be optimized through the standing wave pattern in the composite cavity and spot-area of the spatial modes.
\end{abstract}

Keywords: Semiconductor laser, Mode-locking, Timing jitter, Short pulse generation

\section{INTRODUCTION}

Vertical-cavity surface-emitting lasers (VCSELs) are optical sources that can potentially substitute edge-emitters in many future applications. In particular, vertical-external-cavity surface-emitting lasers (VECSELs) are becoming important devices for high power generation, intracavity frequency conversion, gain-switching and modelocking applications. The external-cavity offers interesting features for mode-locking: good beam quality, the possibility of controlling the transverse laser mode and multi-GHz repetition rates with low tendency to Qswitching. The vertical-external-cavity concept has been applied in optically pumped devices [1-3] and, more recently, to electrically-driven devices $[4,5]$. Optically pumped VECSELs are suited to high power applications because of the scalable output power with the size of the fundamental transverse mode [6]. Optically-pumped VECSELs can be passively mode-locked by semiconductor saturable absorber mirrors (SESAM) [7-9]. They have generated picosecond pulses with output powers larger than $200 \mathrm{~mW}$ and sub-picosecond pulses when operating in the quasi-soliton mode-locking regime $[2,10]$. On the other hand, electrically-driven VECSELs provide more compact laser sources that, after integration of the external cavity, will enable ultrahigh repetition rates [11]. Several demonstrations of electrically-driven VECSELs lasing around $980 \mathrm{~nm}[4,5,12,13]$ have recently been reported. Jasim and coworkers $[5,13]$ considered two distant vertical cavities, an emitter and a saturable absorber, coupled in a linear resonator. Figure 1(a) shows the scheme of the mode-locked VECSEL. The saturable absorber cavity was reverse biased to provided fast recovery of the absorption down to few picoseconds [13]. Jasim et al. found mode-locked pulses with pulse durations of few tens of picoseconds and repetition rates up to $15 \mathrm{GHz}$. Thus far, results from electrically-driven devices suggest that an optimization analysis can be advantageous in order to improve their performance, i.e., to facilitate the mode-locking onset and stability, shorten the pulsewidth

Further author information: (Send correspondence to J. Mulet)

J. Mulet: E-mail: mulet@imedea.uib.es, Telephone: +34 971 172536, Fax: +34 971173426

S. Balle: E-mail: salvador@imedea.uib.es, Telephone: +34 971611733 , Fax: +34 971173426 
(a)

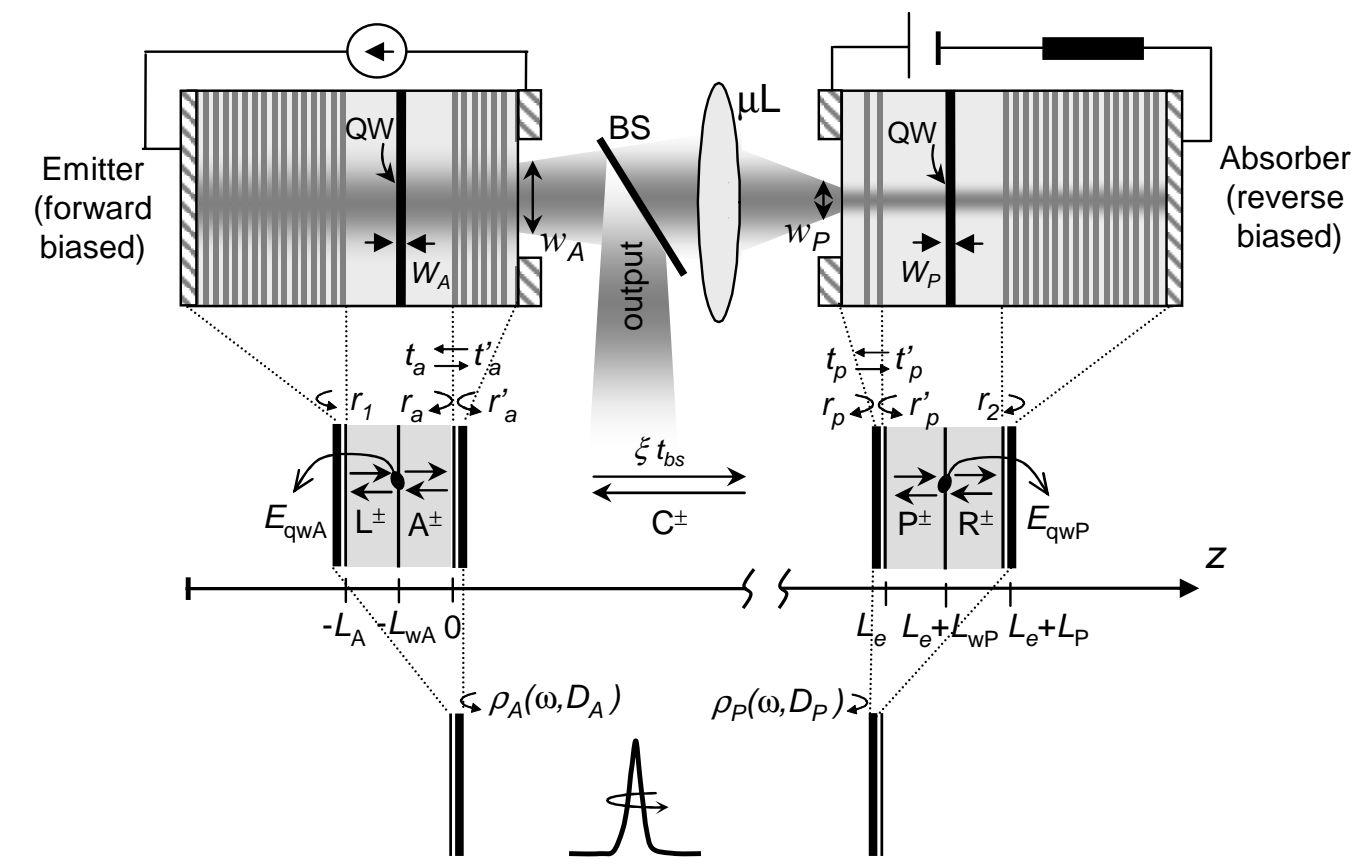

Figure 1. Hierarchy of descriptions. (a) Sketch of the mode-locked VECSEL (not in scale). (b) Partially integrated description: the DBRs are represented as plane mirrors with effective internal (external) reflectivities $r\left(r^{\prime}\right)$ and transmissivities $t\left(t^{\prime}\right)$, beam splitter (BS) and micro-lens $(\mu \mathrm{L})$. (c) Lumped element description: both active cavities are replaced by effective nonlinear reflectivity mirrors $\rho_{a, p}\left(\omega, D_{a, p}\right)$.

and enhance the output power. Moreover, VECSEL demonstrations showed that resonator adjustments are critical to achieve mode-locking $[4,5]$. Self-starting is a problem inherent to fast saturable absorber systems since dynamic gain saturation can stabilize the continuous wave $(\mathrm{CW})$ solution, retarding the appearance of pulsed operation [14]. These experimental results motivated us to develop a detailed theoretical description in view of the optimization of the devices and the generalization of VECSEL concepts to longer wavelengths.

Figure 1 schematically shows three different approaches for modeling the mode-locked VECSEL. Figure 1(a) represents the multi-layer structure that is considered in fully-distributed descriptions of the device. Fully distributed time-domain models of VECSELs provide an accurate description of the intracavity electric fields and the interaction with the active quantum wells (QWs) for ultrafast operation $[15,16]$. Their major drawback, however, is the heavy computational requirements associated with the spatial discretization along the cavity axis, which prevents long integration times, jitter characterization [17], and extensive parametric studies. On the other hand, Fig. 1(c) depicts the starting point of lumped-element models, such as, numerical pulse iterative models $[10,18]$ and Haus master equation [19]. The viewpoint is that of a pulse circulating in the resonator defined by the two vertical cavities. In these models, the vertical cavities are effectively replaced by hard mirrors with nonlinear reflectivity $\rho(\omega, D)$. Therefore, when the pulse encounters a vertical-cavity, the regeneration simultaneously involves amplification/absorption and filtering due to the high finesse of the Fabry-Perot cavities defined by the distributed Bragg reflectors (DBRs). The main difficulty in this approach is the description of the dynamics of the non-linear reflectivities, and two important simplifications are usually made: i) dispersion and nonlinearity are treated separately and lumped into characteristic operators and ii) weak saturation is usually invoked in order to linearize the effective reflectivity. Moreover, tracking the mode-locking onset requires describing a cw solution, and this is a tricky matter in pulse-iterative models because of the finite time window considered around the pulse. Fig. 1(b) depicts an intermediate approach developed by the authors in [20] which, in brief, lumps the DBR layers into hard mirrors with known reflectivity and transmissivity spectra but maintains the detail of the spacers and QWs. Hence, our description assumes that the optical propagation can be solved analytically everywhere in the device except for several thin QW layers. This approach is justified in VCSELs since the the nonlinear 
light-matter interaction solely occurs in the QWs. The benefits of this approach are obvious: it alleviates the complexity of fully-distributed while improving the detail of lumped descriptions. Nonlinear saturation and filtering are naturally accounted for by directly considering the evolution of the intracavity fields at the quantum wells. In addition, no a priori assumptions on either the filtering function or dispersion are required since they naturally arise from the user-defined optical structure. Finally, this partially-integrated approach offers a detailed description of the mode-locking formation which can be tackled via numerical simulations and semi-analytical techniques.

In this paper, we shall discuss the approach followed to derive the partially-integrated model and how it can be used as a tool for optimization, i.e., to find relevant quantities such as mode-locking onset, evaluate the stability, pulsewidth and phase noise.

\section{LASER STRUCTURE}

The mode-locked laser under study is sketched in Fig. 1(a). The structure of the VECSEL is similar to that constructed by Jasim and coworkers in Ref. [5]. It consists of two similar vertical-cavities (emitter and absorber) a distance $L_{e}$ apart. The repetition rate is selected by adjusting the external-cavity length $L_{e}$. The emitter contains InGaAs/GaAsP MQWs at $980 \mathrm{~nm}$, embedded in a $3 \lambda$-cavity defined by two distributed Bragg reflectors. The AlGaAs/GaAs DBRs have a refractive index contrast of $2.967 / 3.525$. The bottom, p-type DBR has high reflectivity $\left(R_{1,2} \sim 0.997\right)$ while the top, n-type DBR has only moderate reflectivity, $R_{a} \sim 0.7-0.8$, and it incorporates a ring-contact that allows for electrical injection (through the substrate). The moderate reflectivity of the top DBR increases the optical losses in the emitter up to the point that it cannot lase by itself unless it receives feedback from the external saturable absorber mirror. Thus, the emitter acts as a Fabry-Perot optical amplifier where the larger the reflectivity the larger the amplified gain at the cost of reducing the available bandwidth. The structure of the saturable absorber section is similar to that of the emitter except for the reflectivity of the top mirror which is approximately that of a semiconductor-air interface $\left(R_{p} \sim 0.3\right)$. The cavity is reverse biased in order to provide fast recovery of the absorption down to few picoseconds [13]. The emitter and the absorber are optically coupled through a micro-lens and a beam splitter, with transmissivity $t_{b s}$, which is used as output coupler. They lie at conjugated planes through the micro-lens, so the spot-area of the spatial beam can be adjusted in order to achieve tighter focusing on the absorber $w_{A} / w_{P}>1$. The angled beam splitter introduces different transmission for TE and TM modes, hence helping to select a linear polarization for the electric field.

\section{A PARTIALLY INTEGRATED MODEL}

The partially integrated model assumes that the optical propagation can be solved analytically in most of the layers composing the VECSEL except for the interaction with the quantum wells which is intrinsically non-linear. The latter shall appear as a nonlinear boundary condition. In order to apply the transfer matrix formalism for propagating fields in the linear layers, we start treating the optical problem in the frequency domain and, thereafter, we invoke the slowly-varying approximation to finally arrive at the "partially-integrated" time-domain model.

\subsection{Optical model in the frequency domain}

As depicted in Fig. 1(a), our system comprises two vertical cavities plus an external cavity. Within the vertical cavities, the wave equation for a linearly-polarized electric field $\mathcal{E}_{\omega}(\vec{r})$ in the frequency domain reads

$$
\nabla^{2} \mathcal{E}_{\omega}(\vec{r})+\frac{\omega^{2}}{c^{2}} n_{s}^{2}\left(\vec{r}_{\perp}, z\right) \mathcal{E}_{\omega}(\vec{r})=-\frac{\omega^{2}}{c^{2} \varepsilon_{0}} \mathcal{P}_{q w}(\vec{r}, \omega)
$$

where $n_{s}^{2}\left(\vec{r}_{\perp}, z\right)=1+\chi_{s}$ stands for the distribution of the background refractive index, and $\mathcal{P}_{q w}(\vec{r}, \omega)$ the distribution of material polarization that is non-vanishing only at the QW layers. The transverse distribution of $n_{s}\left(\vec{r}_{\perp}, z\right)$ arises from thermal lensing and oxide apertures, whereas the $z$-coordinate defines a multilayer structure.

The profile of each transverse mode varies along the VECSEL. To determine the transverse profile, we assume that the micro-lens has a stronger effect defining the spatial mode than the refractive index distribution inside 
the vertical cavities. Therefore, we use as expansion basis the paraxial modes of the external resonator defined by the micro-lens and two frequency selective hard mirrors which represent the two cold-vertical-cavities. The paraxial modes of the VECSEL are determined by Huygen's integral together with the ABCD ray matrices [21]. Following this procedure, we find the distribution of the fundamental Gaussian beam $\Phi_{\omega}\left(r_{\perp}, z\right)$ everywhere in the external cavity. Next, we assume that both the emitter and the absorber are much shorter than the Rayleigh length of the Gaussian beam, so we can neglect diffraction in each of these sections and express the intracavity fields as

$$
\mathcal{E}_{\omega \alpha}(\vec{r})=E_{\omega \alpha}(z) \Phi_{\omega}\left(\vec{r}_{\perp}, z_{\alpha}\right)
$$

where $\alpha=A, P$ stands for the emitter and absorber cavities. Upon introducing (2) into the wave equation (1) and projecting onto $\Phi_{\omega}^{*}\left(\vec{r}_{\perp}, z_{\alpha}\right)$, we arrive at

$$
d_{z}^{2} E_{\omega \alpha}(z)+\frac{\omega^{2}}{c^{2}} n_{\alpha}^{2}(z) E_{\omega \alpha}(z)=-\frac{\omega^{2}}{c^{2} \varepsilon_{0}} \iint \mathcal{P}_{q w \alpha}(\vec{r}, \omega) \Phi_{\omega}^{*}\left(\vec{r}_{\perp}, z_{\alpha}\right) d^{2} \vec{r}_{\perp}
$$

where $n_{\alpha}(z)$ defines the effective index in every layer of the emitter and absorber cavities.

Once the transverse profile is determined for the VECSEL structure, the optical problem reduces to the longitudinal propagation of the optical field given by Eq. (3). Each vertical cavity comprises two DBR layer stacks, two homogeneous spacers and several QW layers. In order to describe these elements, we make the following hypotheses:

1. In a general case, the QWs are distributed along a $m \lambda / 2$ cavity. For simplicity, we assume all the wells identical and in the same electronic state, so that the MQW structure is replaced by a SQW structure with a relative confinement factor scaled by the number of wells. For thin QWs, $W \ll \lambda$, the distribution of material polarization can be approximated by a Dirac's delta

$$
\mathcal{P}_{q w, \alpha}(\vec{r}, \omega)=\varepsilon_{0} \Delta \chi_{\alpha}\left(\omega, N_{\alpha}\right) \mathcal{E}_{\omega, \alpha}\left(\vec{r}_{\perp}, z_{q w}\right) W_{\alpha} \delta\left(z-z_{q w}\right)
$$

$\Delta \chi \equiv \chi(\omega, N)+\chi_{q w}-\chi_{s}, \chi(\omega, N)$ the optical susceptibility of the active QW layer at a carrier density $N\left(\vec{r}_{\perp}\right), \chi_{q w}$ the background refractive index of QWs, $W$ the QW thickness, and $z_{q w}$ the position of the QW in the cavity.

2. Equation (3) becomes linear in the DBRs, so the fields can be analytically propagated in the stack of layers using the transfer matrix formalism [22]. This defines the reflectivity $r_{x}(\omega)$ and transmissivity $t_{x}(\omega)$ spectra of the DBRs. Symbols with prime, $r_{x}^{\prime}(\omega)$ and $t_{x}^{\prime}(\omega)$, will be used to denote external reflectivity and transmissivity, i.e., when a field is injected from the external cavity to a vertical cavity.

3. The spacers at either side of the QW are assumed identical and homogeneous.

Thus far, the problem is solved everywhere in the optical cavity except for the interaction with the QW layers. In the limit of thin QWs, these interaction terms appear as boundary conditions, since Dirac's delta imposes the continuity of the electric field $E_{\omega}(z)$ with a discontinuity of its longitudinal derivative at each QW plane. Upon introducing (4) into (3) and integrating over $z$, we find

$$
d_{z} E_{\omega \alpha}\left(z_{q w}^{+}\right)-d_{z} E_{\omega \alpha}\left(z_{q w}^{-}\right)=-\frac{\omega^{2}}{c^{2}} W_{\alpha} \overline{\Delta \chi_{\alpha}} E_{\omega \alpha}\left(z_{q w}\right)
$$

where $\overline{\Delta \chi}$ stands for the modal optical susceptibility.

It is worth noting that, in this approach, the propagation in all layers is linear everywhere except in the QW, which is treated as an additional boundary conditions by means of (5). This equation imposes a relationship between the field at the QW layer $E_{\omega \alpha}\left(z_{q w}\right)$ and at both spacers. Thus, we solve analytically the longitudinal propagation by expressing the electric field as a set of counter-propagating waves in every section and imposing the 
boundary conditions. See Fig. 1(b) for the notation and Ref. [20] for further details. After some straightforward algebra, the boundary conditions at the emitter can be written in the form

$$
\begin{gathered}
\left(1-r_{1} r_{a} e^{i 2 q L_{a}}\right) E_{q w A}=i c_{A} \Gamma_{A} \overline{\Delta \chi_{A}}\left(\omega, N_{A}\right) E_{q w A}+Y_{A} \\
Y_{A} \equiv t_{a}^{\prime} e^{i q L_{w a}}\left(1+\tilde{r}_{1}\right) C^{-}
\end{gathered}
$$

which only involves the electric field at the QW layer and the externally injected field $Y_{A}$. The meaning of the different parameters is $c_{x}=(\omega / c) M_{x} W_{x} / n_{x}, M_{x}$ the number of QWs, $q=(\omega / c) n_{a}(\omega)$ and $n_{a}$ the effective index of the emitter spacers. The term on the left-hand-side of this equation is the usual Fabry-Perot function that describes multiple pass regeneration and introduces bandwidth filtering. The different parameters are $\tilde{r}_{1}=r_{1} \exp \left(i 2 q\left(L_{a}-L_{w a}\right)\right), \tilde{r}_{a}=r_{a} \exp \left(i 2 q L_{w a}\right)$, and the relative confinement factor $\Gamma_{A}=\frac{1}{2}\left(1+\tilde{r}_{1}\right)\left(1+\tilde{r}_{a}\right)$.

Similarly for the absorber, we arrive at

$$
\begin{gathered}
\left(1-r_{2} r_{p} e^{i 2 k L_{p}}\right) E_{q w P}=i c_{P} \Gamma_{P} \overline{\Delta \chi_{P}}\left(\omega, N_{P}\right) E_{q w P}+Y_{P} \\
Y_{P} \equiv t_{p}^{\prime} e^{i k L_{w p}}\left(1+\tilde{r}_{2}\right) \zeta e^{i \frac{\omega}{c} L_{e}} C^{+}
\end{gathered}
$$

with $k=(\omega / c) n_{p}(\omega)$ and $n_{p}$ the effective index of the absorber spacers. For convenience, $Y_{P}$ is defined as the injection field referenced at the absorber QW plane. The other parameters read $\tilde{r}_{2}=r_{2} \exp \left(i 2 k\left(L_{p}-L_{w p}\right)\right)$, $\tilde{r}_{p}=r_{p} \exp \left(i 2 k L_{w p}\right)$ and $\Gamma_{P}=\frac{1}{2}\left(1+\tilde{r}_{2}\right)\left(1+\tilde{r}_{p}\right)$.

Equations (6)-(9) are the starting point of our optical model. They describe the fields in two Fabry-Perot cavities which experience mutual optical injection. In our case, however, the injection fields are not independent but connected through multipass propagation in the external cavity. The injected fields are expressible as

$$
\begin{aligned}
& Y_{A}(\omega)=t_{b s} \zeta e^{i \frac{\omega}{c} L_{e}}\left[a_{1}(\omega) Y_{P}(\omega)+a_{2}(\omega) E_{q w P}(\omega)\right] \\
& Y_{P}(\omega)=t_{b s} \zeta e^{i \frac{\omega}{c} L_{e}}\left[p_{1}(\omega) Y_{A}(\omega)+p_{2}(\omega) E_{q w A}(\omega)\right]
\end{aligned}
$$

In the external cavity, we take into account localized loss at the position of the beam splitter with transmissivity $t_{b s}$ and $\zeta$ accounts for other distributed losses. The functions $a_{1,2}(\omega)$ and $p_{1,2}(\omega)$ are given in Appendix A. Equations (10)-(11) could be used to eliminate the $Y_{A, P}$ fields in (6)-(8). However, we prefer not to do so since, in the present form, there is a clear separation between internal and external time scales that solely appear in the $\exp \left(i \frac{\omega}{c} L_{e}\right)$ terms of Eqs. (10)-(11). This will be a key point when deriving the time-domain equations.

\subsection{Optical model in the time domain}

In this section, a time-domain version of the frequency-domain model is derived in order to access the modelocking dynamics. A widely used approach is the modal expansion in terms of compound-cavity modes, as performed in frequency-domain theories of mode-locking [23, 24]. However, this expansion may become unpractical when the number of modes is large, as in the present case. Instead, we shall look for an analytical approximation of the Fourier integrals to time-domain. This approach is justified because of the different time scales - the roundtrip times in the vertical cavities and the repetition rate of the external resonator - are clearly separated.

We define the slowly-varying envelope (SVE) of the electric fields with respect to the carrier frequency $\omega_{0}$, $E(t)=\hat{E}(t) \exp \left(-i \omega_{0} t\right)$. We consider $\omega_{0}$ to be the lasing frequency at threshold [20]. The evolution of the electric fields at the QW layers, Eqs. (6)-(9), admits a formal solution in the time-domain that is obtained by replacing $\omega \rightarrow \omega_{0}+i \partial_{t}$, thus accounting for dispersion at all orders. For sufficiently long optical pulses ( $\left.\gtrsim 1 \mathrm{ps}\right)$, the Fabry-Perot functions $\left[1-r_{1}(\omega) r_{a}(\omega) e^{i 2 q(\omega) L_{a}}\right]$ and the optical susceptibility can be expanded in Taylor series around $\omega_{0}$. After Fourier transforming back to time-domain, the SVE of the electric fields $\hat{E}_{q w A, P}$ at the QW layers evolve according

$$
\begin{aligned}
& \kappa_{A} \tau_{r t, A} \partial_{t} \hat{E}_{q w A}(t)=-\left(1-\kappa_{A}\right) \hat{E}_{q w A}(t)+i c_{A} \Gamma_{A} \Delta \chi_{A}\left(\omega_{0}, D_{A}(t)\right) \hat{E}_{q w A}(t)+\hat{Y}_{A}(t)+F_{A}(t) \\
& \kappa_{P} \tau_{r t, P} \partial_{t} \hat{E}_{q w P}(t)=-\left(1-\kappa_{P}\right) \hat{E}_{q w P}(t)+i c_{P} \Gamma_{P} \Delta \chi_{P}\left(\omega_{0}, D_{P}(t)\right) \hat{E}_{q w P}(t)+\hat{Y}_{P}(t)
\end{aligned}
$$


with $\kappa_{A}=r_{10} r_{a 0} e^{i 2 q L_{a}}$, and $\kappa_{P}=r_{20} r_{p 0} e^{i 2 k L_{p}}$. The label "0" means that the quantity is computed at the SVE frequency $\omega_{0}$. A Langevin noise source $F_{A}(t)$ has been phenomenologically added to (12) in order to account for spontaneous emission in the emitter. The (complex) internal roundtrip delays are defined by

$$
\tau_{r t, A} \equiv\left(\tau_{r_{1}}+\tau_{r_{a}}+\frac{2 L_{a}}{v_{g a}}\right)+\frac{c_{A}}{\kappa_{A}} \frac{\partial}{\partial \omega}\left[\Gamma_{A} \Delta \chi_{A}\right]_{\omega_{0}}, \quad \tau_{r t, P} \equiv\left(\tau_{r_{2}}+\tau_{r_{p}}+\frac{2 L_{p}}{v_{g p}}\right)+\frac{c_{P}}{\kappa_{P}} \frac{\partial}{\partial \omega}\left[\Gamma_{P} \Delta \chi_{P}\right]_{\omega_{0}}
$$

$v_{g x}$ being the group velocity in the spacers, and $\tau_{r x}$ the reflection delays of the DBRs. It is worth noting that the last terms in the rhs of the above expressions describe, up to first order, gain and carrier-induced refractive index dispersion. In this way $\tau_{r t, x}$ retain their dependence with the carrier densities, hence describing small timing adjustments through carrier-density fluctuations.

The structure of (12)-(13) resembles to the equations of two semiconductor lasers with mutual optical injection. The terms $\hat{Y}_{A}(t)$ and $\hat{Y}_{P}(t)$ represent the effective injection terms acting onto the QW layers. Their evolution is governed by the iterative equations

$$
\begin{aligned}
& \hat{Y}_{A}(t)=\xi\left[a_{1}\left(\omega_{0}\right) \hat{Y}_{P}\left(t-\tau_{e}\right)+a_{2}\left(\omega_{0}\right) \hat{E}_{q w P}\left(t-\tau_{e}\right)\right] \\
& \hat{Y}_{P}(t)=\xi\left[p_{1}\left(\omega_{0}\right) \hat{Y}_{A}\left(t-\tau_{e}\right)+p_{2}\left(\omega_{0}\right) \hat{E}_{q w A}\left(t-\tau_{e}\right)\right]
\end{aligned}
$$

obtained after Fourier transforming Eqs. (10)-(11). As usual, hat denotes a slowly-varying amplitude, $\xi=$ $t_{b s} \zeta \exp \left(i \omega_{0} L_{e} / c\right)$ is the one-way field attenuation, and $\tau_{e}$ the one-way delay time. The functions $a_{1,2}(\omega), p_{1,2}(\omega)$ are specified in Appendix A. Equations (15)-(16) define an iteration for the injection fields as a function of the delayed memory of the system valid for arbitrarily strong coupling. This effect can be easily numerically implemented by defining vectors that allocate the electric fields at the QWs and the injection fields. In this way, multiple reflections in the external cavity are naturally described. In this sense, the approach presented in this paper is generalizable to other external-cavity configurations involving optical injection and strong optical feedback. Mathematically, the time-domain model is defined as a system of nonlinear ordinary differential equations coupled to an algebraic map for the injection fields.

\subsection{Material model}

We use analytical expressions for the full optical susceptibility (gain and refractive index) given in [25]

$$
\begin{aligned}
\chi_{A}\left(\omega, D_{A},\left|E_{q w A}\right|\right) & =\frac{\Lambda_{A}+1}{2 \Lambda_{A}} f_{A}+\frac{\Lambda_{A}-1}{2 \Lambda_{A}} f_{A}^{*}, \\
f_{A} & =-\chi_{0 A}\left[2 \ln \left(1-\frac{D_{A}}{u_{A}+i \Lambda_{A}}\right)-\ln \left(1-\frac{b_{A}}{u_{A}+i \Lambda_{A}}\right)\right],
\end{aligned}
$$

with $\Lambda_{A}=\sqrt{1+\epsilon_{A}\left|E_{q w A}\right|^{2}}, \epsilon_{A}$ the gain compression coefficient due to spectral-hole burning, and the normalized frequency $u_{A}=\left(\omega-E_{g A} / \hbar\right) / \gamma_{2 A}+\sigma\left(D_{A}\right)^{1 / 3} . \sigma$ phenomenologically describes bandgap renormalization with the carrier density which has been normalized to the transparency value $D_{A} \equiv N_{A} / N_{t A}$. Finally, $\gamma_{2 A}$ stands for the material polarization dephasing-rate. The value of $\chi_{0 A}$ is taken as a free parameter to achieve a material gain of $1500 \mathrm{~cm}^{-1}$ per well at $D_{A} \approx 2$. The full optical susceptibility allows for an efficient description of the nonlinear gain saturation and self-phase modulation effects at time scales above one picosecond.

In the saturable absorber, the expressions for the optical susceptibility are equivalent to Eq. (17) except for $u_{P}=\left(\omega-E_{g P} / \hbar\right) / \gamma_{2 P}$. Due to the quantum confined Stark effect in InGaAs/GaAsP QWs, the bandgap $E_{g P}$ experiences a redshift of $\sim 5 \mathrm{meV}$ per applied volt in reverse bias [26].

A final point consists in determining the carrier densities populating the wells. In order to find a simple and efficient description, the transverse spatial dependence is eliminated by assuming homogeneous saturation energies in regions defined by the mode areas. As already mentioned, this approximation is justified when the transverse profiles are mainly defined by the micro-lens and the influence of Kerr and thermal lensing effects is negligible. The evolution equations for the spatially-averaged carrier densities, $D_{x}=\bar{N}_{x} / N_{t x}$ read [27]

$$
\begin{aligned}
\gamma_{e}^{-1} \partial_{t} D_{A} & =\mu-D_{A}^{2}+\frac{1}{2 i}\left[\mathcal{P}_{A}(t) E_{q w A}^{*}(t)-c . c .\right] \\
\gamma_{e}^{-1} \partial_{t} D_{P} & =-\gamma_{a b s} D_{P}+s \frac{1}{2 i}\left[\mathcal{P}_{P}(t) E_{q w P}^{*}(t)-c . c .\right]
\end{aligned}
$$


where the material polarization after a first-order expansion around $\omega_{0}$ reads [27]

$$
\mathcal{P}_{\alpha}(t)=\frac{1}{2 \pi} \int_{-\infty}^{+\infty} d \omega \chi_{\alpha}\left(\omega, D_{\alpha}\right) E_{q w \alpha}(\omega) e^{-i \omega t} \approx \chi_{\alpha}\left(\omega_{0}, D_{\alpha}(t)\right) \hat{E}_{q w \alpha}(t)+i \partial_{\omega} \chi_{\alpha}\left(\omega_{0}, D_{\alpha}(t)\right) \partial_{t} \hat{E}_{q w \alpha}(t) .
$$

Note that the electric fields and carrier densities are expressed in dimensionless units and that non-radiative recombination at $980 \mathrm{~nm}$ has been neglected. The meaning of the different parameters is $\gamma_{e} \equiv B_{e f f} N_{t A}$, $B_{e f f}$ is the effective spontaneous recombination constant [28], and $\gamma_{a b s} \equiv 1 /\left(\gamma_{e} \tau_{a b s}\right) . \quad \tau_{a b s}$ is the absorber recovery time obtained from fitting of the experimental data [13], $\tau_{a b s}\left(V_{a b s}\right)=21 \mathrm{ps} \cdot \exp \left(V_{a b s} / 2.125 \mathrm{~V}\right)$ for $V_{a b s} \in[-4,0] \mathrm{V}$. The factor $s$ that multiplies the stimulated recombination term in the absorber equation reads $s \equiv\left(n_{a} N_{t A} w_{A}^{2}\right) /\left(n_{p} N_{t P} w_{P}^{2}\right)$, with $w_{x}$ the $1 / e$-beam waists of the modes in the each cavity. The scaled current injected into the emitter is $\mu \equiv I /\left(e M_{q w} W_{A} A_{\perp} B_{r a d} N_{t A}^{2}\right)$, $e$ the elemental charge, $M_{q w} W_{A}$ the total thickness of QWs, and $I$ the total injected current. The details of the current distribution, that should be obtained by solving the electrical conduction equation, only define the effective active area in the emitter $A_{\perp}$, thus the current density and heating.

\subsection{Compound-cavity modes}

Continuous wave $(\mathrm{cw})$ operation is the simplest solution of the time-domain model; the compound-cavity modes (CCM) represent monochromatic solutions for the electric field and steady-state inversions. The CCM can be expressed as follows

$$
\hat{E}_{q w, x}(t)=E_{q w x}^{s} e^{-i \omega_{s} t}, \quad \hat{Y}_{x}(t)=Y_{x}^{s} e^{-i \omega_{s} t}, \quad D_{x}(t)=D_{x}^{s} .
$$

Upon introducing these expressions into the time-domain model (12)-(13), (15)-(16) and eliminating the dependence on injection fields $Y_{x}$, we arrive at two equations for the fields at the QW planes coupled to equations (18)-(19) for the stationary carrier densities. The field equations read

$$
\begin{aligned}
& {\left[\tilde{N}_{11}\left(\omega_{s}\right)-i \omega_{s} \tau_{r t A}^{s}-i c_{A} \Gamma_{A} \Delta \chi_{A}\left(\omega_{0}, D_{A}^{s}\right)\right] E_{q w A}^{s}=\tilde{N}_{12}\left(\omega_{s}\right) E_{q w P}^{s}} \\
& {\left[\tilde{N}_{22}\left(\omega_{s}\right)-i \omega_{s} \tau_{r t P}^{s}-i c_{P} \Gamma_{P} \Delta \chi_{P}\left(\omega_{0}, D_{P}^{s}\right)\right] E_{q w P}^{s}=\tilde{N}_{21}\left(\omega_{s}\right) E_{q w A}^{s}}
\end{aligned}
$$

where the notation $\tau_{r t x}^{s}=\tau_{r t x}\left(D_{x}^{s}\right)$ has been introduced. The coefficients of the $\tilde{N}_{i j}$ matrix are defined in Appendix B. A nontrivial solution for $E_{q w x}^{s}$ in (22)-(23) requires the condition of vanishing characteristic determinant of the system. This provides a complex equation $\mathcal{D}\left(\omega_{s}, D_{A}^{s}, D_{P}^{s}\right)=0$ with three real unknowns. To solve this equation we consider two cases. CCM can be classified into on-state and off-state depending on whether the saturable absorber is partially bleached or not, respectively.

\subsubsection{OFF-CCMs}

The off-state CCMs are obtained by neglecting the stimulated recombination terms in the carrier density equations. Moreover, $D_{P}^{s} \approx 0$ since the absorber is unbleached. Therefore, the characteristic equation reduces to $\mathcal{D}\left(\omega_{s}, D_{A}^{s}=\sqrt{\mu_{s}}, D_{P}^{s}=0\right)=0$ which can be solved for $\omega_{s}$ and $D_{A}^{s}$. This leads to two real nonlinear equations whose roots are located by contour plotting and numerically solved with a two-dimensional Newton-Raphson scheme. The stability of these modes is simply determined by the threshold condition, being stable (unstable) for $\mu<\mu_{s}\left(\mu>\mu_{s}\right)$. The lowest $\mu_{s}$ defines the laser threshold current $I_{t h}$ and it also determines the emission frequency of the laser at threshold.

\subsubsection{ON-CCMs}

Above the laser threshold, an additional equation is required in order to determine the bleaching level in the absorber. The remaining condition is obtained from the carrier equations, which impose a ratio for the stimulated recombination terms [29]

$$
\left|\frac{E_{q w A}^{s}}{E_{q w P}^{s}}\right|^{2}=\frac{-\left(\mu-D_{A}^{s}{ }^{2}\right)}{\gamma_{a b s} D_{P}^{s}} s \frac{\operatorname{Im}\left[\chi_{P}^{s}+\chi_{P}^{s}{ }^{\prime} \omega_{s}\right]}{\operatorname{Im}\left[\chi_{A}^{s}+\chi_{A}^{s}{ }^{\prime} \omega_{s}\right]}=\frac{\left|\tilde{N}_{12}\left(\omega_{s}\right)\right|^{2}}{\left|\tilde{N}_{11}\left(\omega_{s}\right)-i \omega_{s} \tau_{r t A}^{s}-i c_{A} \Gamma_{A} \chi_{A}^{s}\right|^{2}}
$$


where $\chi_{x}^{s}=\chi_{x}\left(\omega_{0}, D_{x}^{s}\right)$ and $\chi_{x}^{s \prime}=\partial \chi_{x}\left(\omega_{0}, D_{x}^{s}\right) / \partial \omega$. The procedure followed to solve these equations is the following. First, a convenient way to locate the on-CCMs is by recalling that each off-mode will have an associated on-CCM for currents beyond the laser threshold. In practice, $\omega_{s}$ changes little with respect to the associated off-CCM. Second, we consider $\mathcal{D}\left(\omega_{s}, D_{A}^{s}, D_{P}^{s}\right)=0$ as an equation for $\omega_{s}\left(D_{P}\right)$ and $D_{A}\left(D_{P}\right)$ which have a parametric dependence on $D_{P}$. Thereafter, Eq. (24) is solved for $D_{P}$. An on-CCM is characterized by the quantities $\left\{\omega_{s}, D_{A}^{s}, D_{P}^{s},\left|E_{q w A}^{s}\right|,\left|E_{q w P}^{s}\right|, \Psi_{A P}\right\}$, i.e., emission frequency $\omega_{s}$ (with respect to $\omega_{0}$ ), inversions, the field amplitudes, and a relative phase between the fields.

\subsubsection{Linear stability analysis of on-CCMs}

In order to perform the linear stability analysis (LSA) of a given on-CCM, we add a small perturbation to the monochromatic electric field and steady carrier densities that represents excitation of adjacent longitudinal modes

$$
\begin{aligned}
\hat{E}_{q w, x}(t) & =E_{q w x}^{s} e^{-i \omega_{s} t}+\delta E_{q w x} e^{-i\left(\omega_{s}+i \lambda\right) t} \\
\hat{Y}_{x}(t) & =Y_{x}^{s} e^{-i \omega_{s} t}+\delta Y_{x} e^{-i\left(\omega_{s}+i \lambda\right) t} \\
D_{x}(t) & =D_{x}^{s}+\Delta_{x} e^{\lambda t}
\end{aligned}
$$

Upon introducing these expressions into the dynamical model and linearizing the perturbations, we find a $6 \times 6$ homogeneous system for $\delta E_{q w A}, \delta E_{q w A}^{*}, \delta E_{q w P}, \delta E_{q w P}^{*}, \Delta_{A}$ and $\Delta_{P}$. We arrive at a complex equation for the eigenvalue $\lambda=\lambda_{R}+i \lambda_{I}$. A net growth rate of the perturbations, $\lambda_{R}>0$, indicates that such on-mode is unstable and, depending on the conditions, mode switching or multimode dynamics will develop. The resulting transcendent equation has a large number of solutions associated with the multiple resonances of the system. For mode-locking one is interested in looking for roots with imaginary part close to $\lambda_{I} \approx \Omega \cdot(0, \pm 1, \pm 2, \cdots)$, with $\Omega$ the inter-mode spacing. In particular, fundamental mode-locking involves excitation of neighboring modes with $\lambda_{I} \approx \pm \Omega$.

\subsection{Parameters}

The different parameter values used in the model are taken to reproduce the experimental conditions in Ref. [5]. For both the emitter and the absorber, the reference Bragg wavelength is $\lambda_{B}=980 \mathrm{~nm}$, while the reflectivity of bottom DBRs (23 pairs) is $R_{2}=R_{1}=0.998$. The reflectivity of the top DBR in the emitter is $R_{a} \sim 60-80 \%$ $\left(\mathbb{P}_{A} \sim 3-5\right.$ pairs $)$ while in the absorber we take $R_{p} \sim 30 \%$ ( $\mathbb{P}_{P} \sim 1-2$ pairs). The modal refractive indexes are $n_{a}=n_{p}=3.52$, and the group refractive index is $n_{g}=4.5$. The QW thickness is $W_{A}=W_{P}=9 \mathrm{~nm}$, and the number of quantum wells $M_{q w A}=4$ and $M_{q w P}=1-4$. The susceptibility pre-factor is $\chi_{0 A}=\chi_{0 P}=0.1$, the empty band contribution to the susceptibility in the emitter is $b_{A}=10^{4}$ and in the absorber $b_{P}=2 \cdot 10^{4}$. The gain suppression coefficient $\epsilon_{A}=\epsilon_{P}=10^{-23} \mathrm{~m}^{-3}$, the reference bandgap for the gain in the emitter $E_{g A}=1.265$ $\mathrm{eV}$ and in the absorber $E_{g P, \mathrm{ref} f}=1.285 \mathrm{eV}$, the reverse bias in absorber $V_{a b s}=-3 \mathrm{~V}$, the polarization dephasing rate in the emitter $\gamma_{2 A}=2 \cdot 10^{13} \mathrm{~s}^{-1}$ and in the absorber $\gamma_{2 P}=10^{13} \mathrm{~s}^{-1}$, the bandgap shrinkage coefficient in the emitter $\sigma=0.2$, the effective spontaneous recombination constant $B_{\text {eff }}=1.5 \cdot 10^{-16} \mathrm{~m}^{3} / \mathrm{s}$, the carrier density at transparency in the emitter $N_{t A}=1.5 \cdot 10^{24} \mathrm{~m}^{-3}$ and in the absorber $N_{t P}=0.75 \cdot 10^{24} \mathrm{~m}^{-3}$. Finally, the effective active region area is $A_{\perp} \sim \pi \cdot 75^{2} \mu \mathrm{m}^{2}$, and the beam waist ratio is $w_{A} / w_{P} \sim 2$. In order to operate close to the Bragg wavelength, the cavity lengths $L_{x}$ fulfill the usual resonance condition in a $3 \lambda$-cavity [28].

\section{RESULTS}

\subsection{General considerations}

In the emitter, the detuning between the Bragg and bandgap frequencies is set to $\Delta=\left(\omega_{B}-E_{g A} / \hbar\right) / \gamma_{2 A} \approx 1$ in order to operate close to the peak of maximum gain at typical carrier densities $D_{A} \approx 2-3$. In the absorber, the nominal bandgap and the reverse bias are adjusted in order to obtain similar saturation fluences in the emitter and absorber. Hence, the operation in the absorber occurs around the absorption edge although not too far from the bandgap, yielding moderate absorption and positive linewidth enhancement factors. The typical values we obtain are $\alpha_{A} \approx 2-3$ in the emitter and $\alpha_{P} \approx 0-1$ in the absorber. 
$(\alpha)$

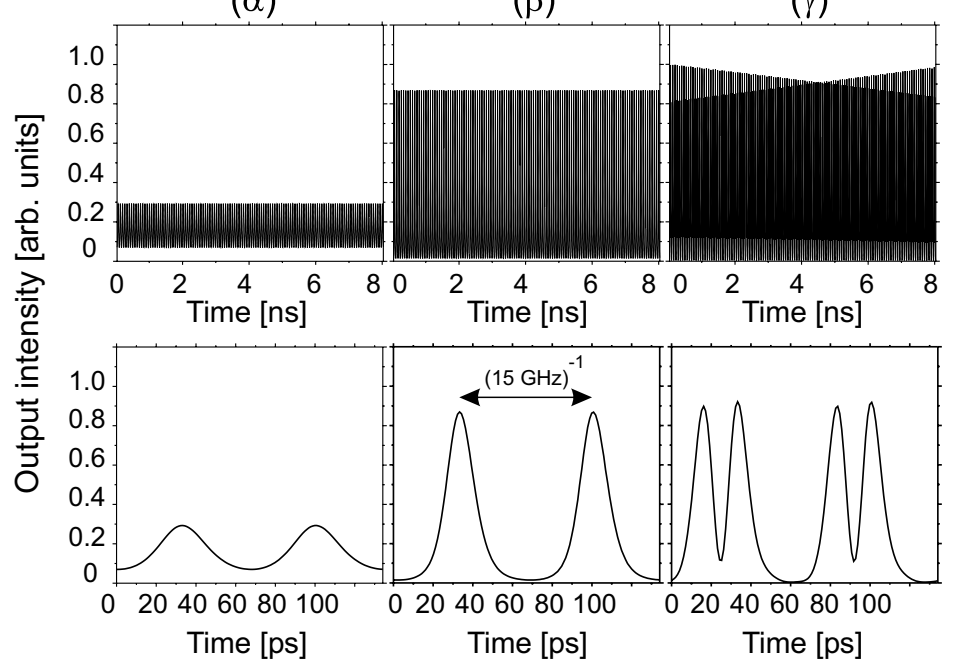

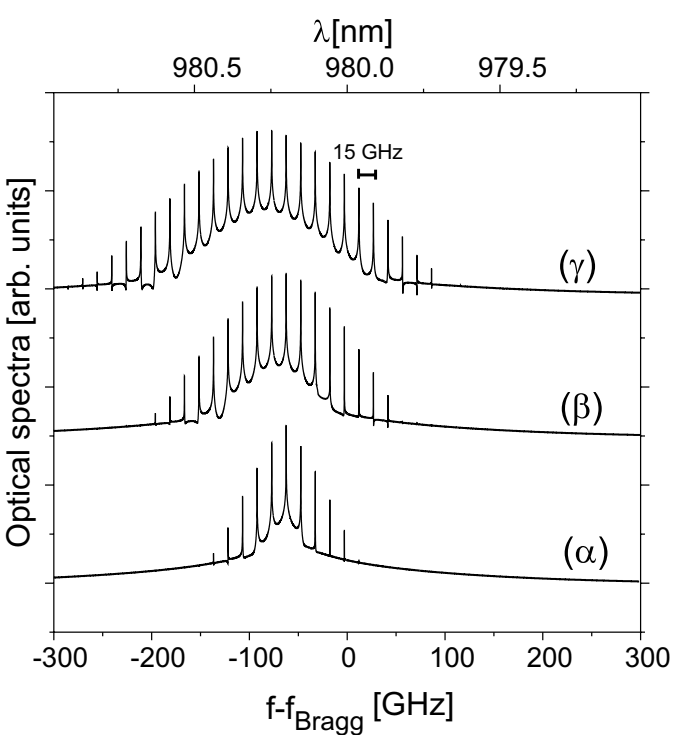

Figure 2. (Left panel): Pulse train for different injection currents normalized to the laser threshold $I=1.2 I_{t h}(\alpha)$, $I=1.3 I_{t h}(\beta)$ and $I=1.5 I_{t h}(\gamma)$. We go from oscillations, stable pulses to incomplete mode-locking. (Right panel): Optical spectra for the same conditions. The plots have been vertically shifted for the sake of clarity. Parameters are in Sec. 3.5 except for: $t_{b s}=0.9, s=8, \mathbb{P}_{A}=4$ and $\mathbb{P}_{P}=1, M_{q w A}=4$ and $M_{q w P}=1$.

The (differential) saturation energy of the active media can be expressed as $E_{s a t, x}\left(\omega_{0}, N_{x}\right)=\pi w_{x}^{2} F_{s a t, x}$, $F_{\text {sat }, x}$ being the saturation fluence or saturation per unit area. The ratio of saturation energies $E_{\text {sat }, A} / E_{\text {sat }, P}$ can be controlled by adjusting the aspect of mode beam waists $w_{x}$ with the micro-lens. We will show that a crucial condition to achieve mode-locking is the prior adjustment of the mode sizes. In particular, the mode has to be more tightly focused on the absorber facet. In addition, the relative intensities of the standing-wave patterns within each vertical-cavity plays an important role in defining the mode-locking onset.

\subsection{Scenario upon increasing the injection current}

Figure 2 shows three simulated pulse trains for different injection levels in the emitter. The repetition rate is set to $15 \mathrm{GHz}$ as in the experiments reported in Ref. [5]. The initial transients that appear after each change in bias current have been removed. The general scenario is the following. When setting the bias current sufficiently close to the laser threshold, the VECSEL emits $\mathrm{cw}$ in a single longitudinal mode. Increasing the bias current, the output intensity undergoes harmonic oscillations at a frequency near the inverse of the cavity roundtrip time. This fact is associated with the activation of longitudinal side modes. The optical spectrum [Fig. $2(\alpha)$ ] shows several longitudinal modes. This stage is followed by a rapid onset of multi-longitudinal mode emission. A frequency comb, characteristic of mode-locking, develops in the different optical spectra. In the time-domain, the harmonic oscillations reshape into pulses when the current is increased. For certain injection currents beyond threshold, mode-locking may enter into an irregular region as shown in Fig. $2(\gamma)$. In simulations, this instability leads to pulse splitting and a slow ( $100 \mathrm{~ns})$ modulation of the pulse train. Experimentally, there is evidence that the laser can display a similar unstable self-pulsating behavior [5]. At this stage, the quantities characterizing the pulse, such as the pulse energy, become time dependent. The instability point can be moved to higher currents by increasing the reverse bias applied to the absorber up to a maximum value around $-4 \mathrm{~V}$.

\subsection{Tracking the mode-locking onset}

In order to better understand the scenario presented in the preceding section, we track the onset of the modelocked state by means numerical simulations of the time-domain model and semi-analytical techniques based on the stability analysis of compound-cavity modes (CCMs).

The numerical simulations in the previous section indicate that the mode-locked state develops in a continuous way soon after the monochromatic solutions lose stability. Thus, the onset of mode-locking can be characterized 

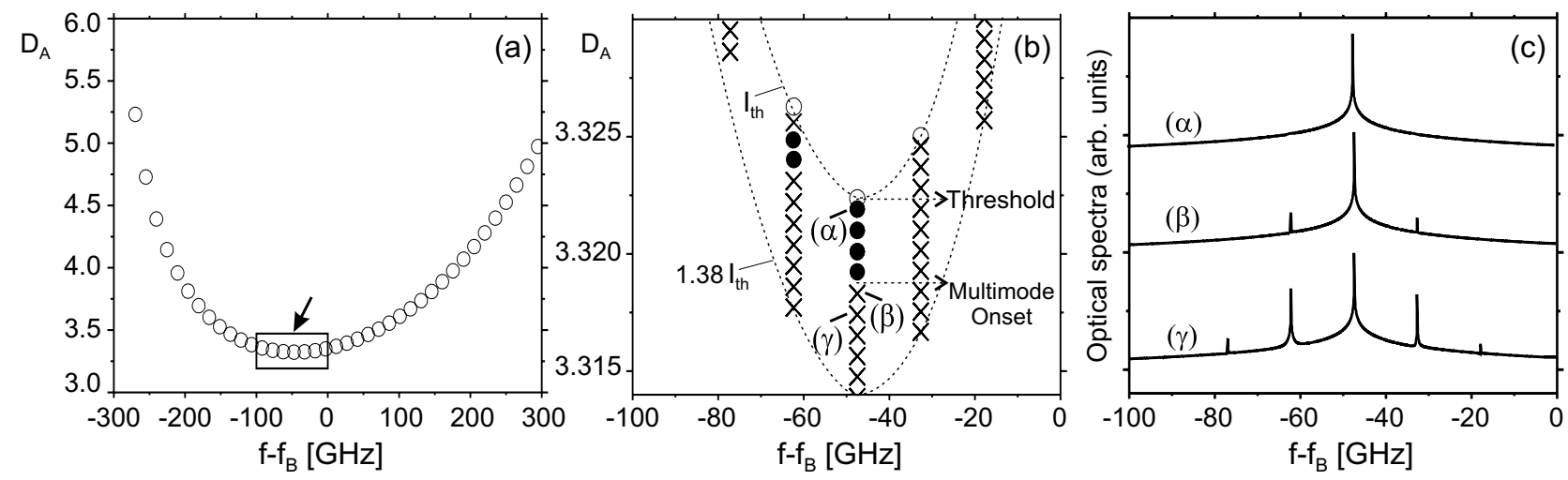

Figure 3. (a) Open circles depict off-CCMs inversion and frequency with respect to the Bragg resonance. (b) Stable (•) and unstable $(\times)$ on-modes. The on-modes are calculated for injection levels ranging from $1.02 I_{t h}$ to $1.38 I_{t h}$ at $0.04 I_{t h}$ steps (from top to bottom). (c) Optical spectra for three different currents. Same conditions as in Fig. 2.

by analyzing the linear stability of the CCMs, which are the monochromatic solutions of the electric field and steady-state inversions. As discussed in Sec. 3.4, CCMs can be classified into on-state and off-state depending on whether the saturable absorber is partially bleached or not, respectively. Empty circles in Fig. 3(a) show the inversion level in the emitter required for the different off-CCMs to become unstable, ordered according to the modal frequency in these working condition. The separation between nearest modes $\Omega(15 \mathrm{GHz})$ is determined by the external cavity length $\left(L_{e} \approx 1 \mathrm{~cm}\right)$. The lowest inversion off-CCM defines the laser threshold. The inversion needed for an off-CCM become unstable increases when its frequency deviates from the threshold mode, thus limiting the available bandwidth. This effect stems from the multiple-pass regeneration in vertical cavities, where the larger the amplified gain the lower the optical bandwidth. The optical bandwidth can be enhanced by reducing the number of pairs of the top DBRs, thus supporting shorter pulses at the expense of increasing the threshold current. For injection currents above the laser threshold, the on-CCMs have optical power that partially bleaches the saturable absorber, reducing the overall cavity losses. Therefore, their inversion condition is below that of the associated off-CCM and, the larger the injection level in the emitter the larger the inversion reduction [Fig. 3(b)]. In order to facilitate the bleaching of the absorber, low finesse in the emitter and moderate finesse in the absorber cavities are recommended.

The stability of the different CCMs above threshold is determined performing the linear stability analysis (LSA) described in Sec. 3.4.3. Operation in a given on-mode is perturbed with respect to excitation of neighboring modes, arriving at a transcendent equation for the eigenvalue $\lambda$. A sufficient condition for multimode emission occurs when no on-state mode is stable against excitation of adjacent longitudinal modes. Figure $3(\mathrm{~b})$ zooms into a region near the threshold mode. It shows an example of mode-locking onset calculated for $\mathbb{P}_{A}=4\left(\mathbb{P}_{P}=1\right)$ pairs in the top DBR of the emitter (absorber) cavity. We simultaneously follow the CCMs, LSA, temporal and spectral properties [Fig. 3(c)] upon increasing the injection level in the emitter. The stable (unstable) on-CCM are marked with circle (cross) symbols. When setting the bias current sufficiently close to the laser threshold $I_{t h}$, the VECSEL emits cw in the stable on-CCM associated with the threshold mode. The optical spectrum in Fig. 3(c) labeled as $(\alpha)$ shows this single mode active. For certain current intervals, CCMs placed to the red of the threshold mode are also stable leading to multi-stability. Nonetheless, numerical simulations indicate that the system keeps operating on the on-threshold mode. This on-mode loses stability at $I \approx 1.15 I_{t h}$ where no other CCM is stable. This point sets the locus for multi-longitudinal-mode emission which reflects itself as the excitation of side modes in the optical spectrum [Fig. 3(c) label $(\beta)$ ] and harmonic intensity oscillations in the time-domain [Fig. 2( $\alpha$ )]. Hence, LSA correctly determines the onset of multimode emission according to the results from numerical simulations. It is also clear that these oscillations are because of multimode dynamics since they appear at the external cavity frequency. According to the frequency-domain theories of mode-locking [23], mode-locking is likely to occur when a multimode solution sets in. Since, conventional continuation methods are out of the scope for our dynamical system, we use numerical simulations to verify the stability of the pulsed solution. Numerical simulations demonstrate that fully-developed mode-locked pulses appear when the injection 
current in the emitter is further increased beyond the multimode onset. This is a characteristic feature of the bandwidth limitation introduced by the vertical cavities. For larger injection levels in the emitter, many modes participate in the dynamics yielding a frequency comb in the optical spectra, and the harmonic oscillations rapidly reshape into pulses.

\subsection{Optimization analysis}

In order to complete the characterization of the mode-locking onset, we have undertaken extensive numerical simulations of the time-domain model. In this way, the effects of the spot-area ratio, finesse of the cavities and number of quantum wells on the mode-locking onset are investigated.

The diagrams of Fig. 4 demonstrate the appearance of different regimes of operation when the abovementioned parameters are varied. At fixed spot-area ratio, the mode-locking onset with current is similar to the one discussed in Fig. 2: we go from cw operation to fully-developed pulses through intensity oscillations. Circle symbols in the figure show the locus for multimode onset determined from LSA which agrees with the appearance of oscillations in simulations. These oscillations can be regarded as mode-locking of a small number of modes. However, the harmonic regime may be unappropriate for applications. Therefore, we use a criterium for fully-developed mode-locked (FDML) pulses occurring when the suppression between the first and second harmonic peaks in the power spectrum is less than $3 \mathrm{~dB}$, providing sufficiently nonharmonic oscillations. Regions of FDML, indicated in Fig. 4, appear beyond the multimode onset. Another salient feature is that the mode-locking onset is favored by more tightly focusing the spatial mode on the absorber facet. In fact, there exists a critical value of the spot-area ratio to obtain FDML close-to-threshold where the fundamental transverse mode performance is maintained. In devices with integrated absorbers, the spot areas in the emitter and absorber cavities are essentially equal, thus the contrast in saturation energies must be obtained by designing absorbers with low saturation fluences. This can be accomplished with quantum dot saturable absorbers [11].
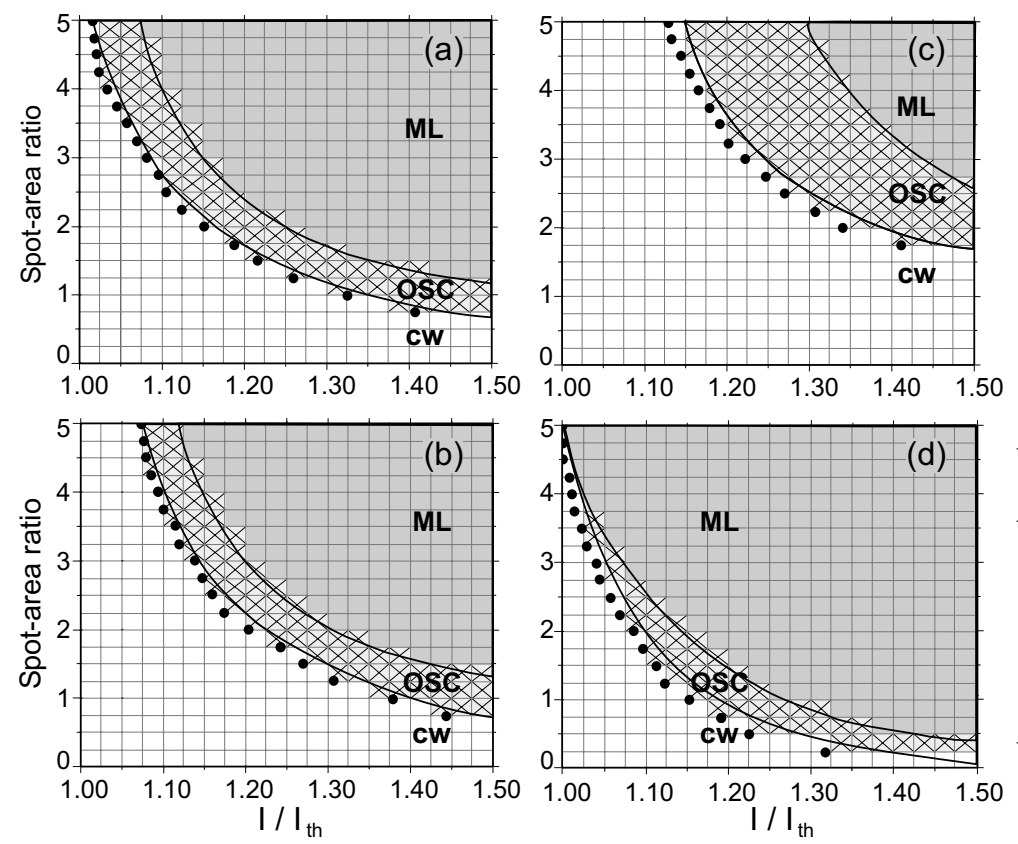

\begin{tabular}{ccc}
\hline Pairs & Reflectivity & $\begin{array}{c}\text { Reflection } \\
\text { delay (fs) }\end{array}$ \\
\hline 1 & 0.4436 & 2.460 \\
2 & 0.5634 & 4.203 \\
3 & 0.6670 & 5.462 \\
4 & 0.7507 & 6.373 \\
5 & 0.8162 & 7.031 \\
6 & 0.8659 & 7.504 \\
\hline
\end{tabular}

Figure 4. Mode-locking regimes with the spot-area ratio and injection current: continuous wave (cw), oscillations (OSC), and fully-developed mode-locked pulses (ML). Circle symbols are the analytical predictions from LSA. All panels are for $4 \mathrm{QW}$ in the emitter and $1 \mathrm{QW}$ in the absorber except for panel (d) that is for $4 \mathrm{QWs}$. The number of pairs in the top DBR of the emitter and absorber is (a) $\mathbb{P}_{A}=3, \mathbb{P}_{P}=1$; (b) $\mathbb{P}_{A}=4, \mathbb{P}_{P}=2$; (c) and (d) $\mathbb{P}_{A}=4, \mathbb{P}_{P}=1$. The equivalence between reflectivity and number of pairs of top DBRs is given in the table. 

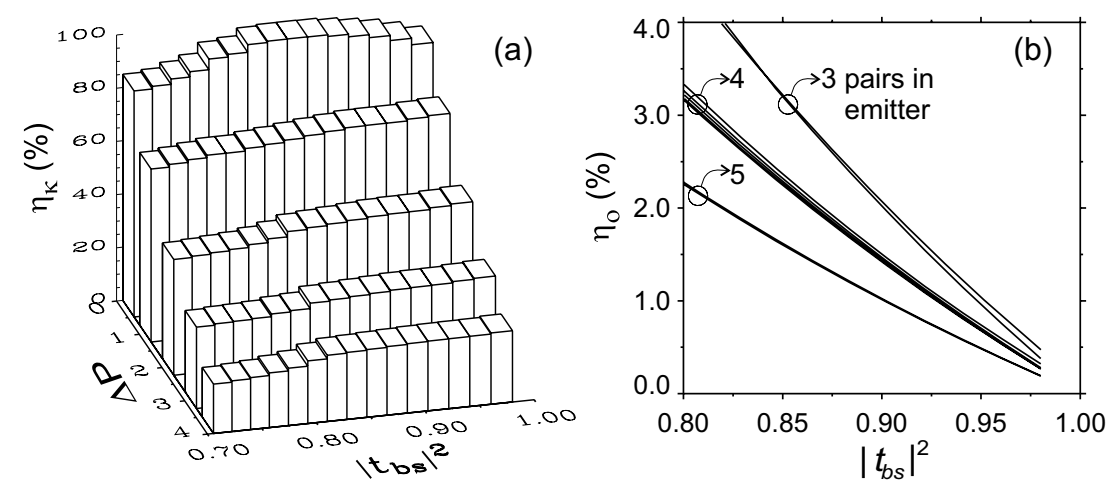

Figure 5. (a) Coupling and (b) output efficiency for different beam splitter transmissivity and finesse of the cavities.

The finesse of the vertical cavities has a strong effect defining the stability of the modes and, in turn, the mode-locking onset. We find that when the absorber is more effectively bleached, the stability range of the on-modes is narrower and the multimode onset appears earlier. Panels in Fig. 4 correspond to different finesse of the vertical cavities and number of QWs. The coupler transmissivity has been adjusted to compensate for inversion variations, thus keeping constant the saturation fluences. In vertical cavities, the saturation energies can also be controlled through their finesse which defines the standing-wave pattern in the composite cavity. We define the coupling efficiency as the ratio of the standing-wave pattern intensities at the QW layers of the absorber and emitter, $\eta_{\kappa} \equiv\left|E_{q w P} / E_{q w A}\right|^{2}$, computed at threshold conditions for the inversion and frequency. Fig. 5(a) shows that the coupling efficiency slightly increases with $t_{b s}$. A figure of merit, controlling the coupling strength, is the difference in number of pairs of top DBRs $\Delta \mathbb{P}=\mathbb{P}_{A}-\mathbb{P}_{P}$ between the emitter and absorber. The lower (higher) the finesse of the emitter (absorber) the larger the coupling, approaching $100 \%$ for symmetric cavities. Hence, the mode-locking onset is favored in Fig. 4(a) and (b) where $\eta_{\kappa} \approx 0.5(\Delta \mathbb{P}=2)$ compared with the panel (c) that only has $\eta_{\kappa} \approx 0.35(\Delta \mathbb{P}=3)$.

In the last example [Fig. 4(d)], the onset is favored by increasing the number of QWs in the absorber owing to the larger modulation depth of the nonlinear absorber reflectivity. However, in this case, the system has to be operated at higher $t_{b s}$ to maintain the threshold current which reduces the output power. The output efficiency provides the ratio between the output power and the intracavity optical power in the emitter $\eta_{o} \equiv\left|E_{\text {out }} / E_{q w A}\right|^{2}$. Therefore, there exists a trade-off between threshold current and output power since higher $t_{b s}$ results into smaller output efficiency [Fig. 5(b)].

\subsection{Pulsewidth and Phase noise}

The dependence of the pulsewidth on the injection current is shown in Fig. 6(a). For well developed pulses, the pulsewidth is of the order of $10 \mathrm{ps}$ in agreement with experimental findings [5]. The pulses slightly shorten when the injection current increases. In practice, there exists a maximum injection current accessible for fundamental transverse mode operation. Fig. 6(a) demonstrates that the pulsewidth can be shortened by reducing the reflectivity of the top DBR in the emitter or increasing the number of quantum wells in the absorber, the fact that also facilitates the mode-locking onset. However, there is a trade-off between gain and bandwidth that has to be optimized in order to maintain the threshold characteristics of the device. The pulses are slightly chirped due to the combined effect of self-phase modulation effects and cavity dispersion, leading to a spectral broadening of the pulses. The typical time-bandwidth product computed for these pulses is $\sim 0.5$.

Figure 6(b) shows the phase-noise spectrum computed for three different injection currents using the techniques outlined in Ref. [17]. The phase noise spectrum provides the noise skirts from $100 \mathrm{kHz}$ (maximum integration time) up to Nyquist. For passive mode-locking, there exists a $1 / \Omega^{2}$ noise decay typical of passive mode-locking. Amplitude noise is strongly suppressed at $\mathrm{GHz}$ frequency range. Only in the frequency range 1-10 MHz, several peaks can be identified which are a signature of slow amplitude fluctuations. The amplitude of these peaks increase when entering into the incomplete mode-locking region. The slow envelope of the pulse train 

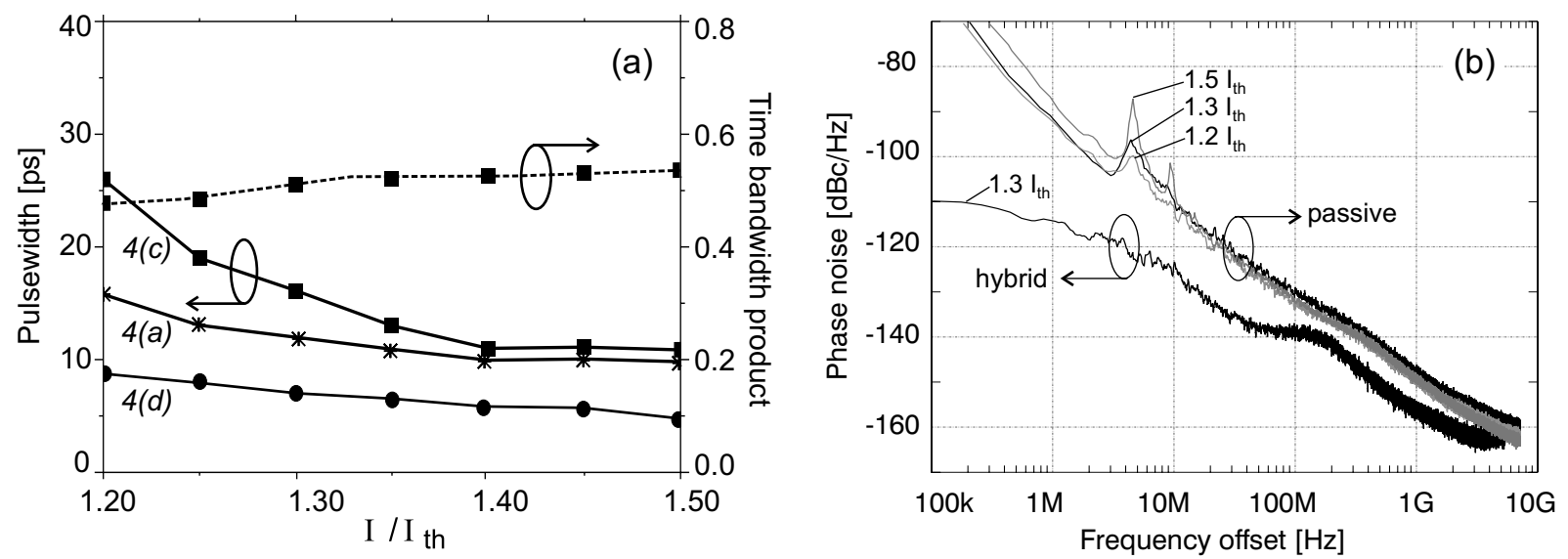

Figure 6. (a) Uncompressed pulsewidth and time bandwidth product as function of the injection current. The parameters are those of Fig. 4(c) for ( $\mathbf{\square})$, Fig. 4(a) for $(*)$, and Fig. 4(d) for $(\bullet)$. The spot-area ratio is fixed to $w_{A} / w_{P}=4$. (b) Phase noise spectrum for three different injection currents $1.2 I_{t h}, 1.3 I_{t h}$ and $1.5 I_{t h}$ for the conditions in (ם). For hybrid mode-locking, a sinusoidal modulation with amplitude of $1 \mathrm{~V}$ and frequency $f_{m} \approx 15 \mathrm{GHz}$ is added to the absorber voltage.

corresponds to this frequency [Fig. 2( $\gamma)$ ]. Phase noise spectrum can be substantially reduced at low frequency offsets by adding a sinusoidal modulation to the reverse bias in the absorber. In addition, hybrid mode-locking is convenient since leads to shorter pulses and better extinction ratio of the pulses. Synchronization locking by reverse voltage modulation is demonstrated which opens the possibility of using these devices as precise emitters and for clock recovery applications.

\section{DISCUSSION AND CONCLUSIONS}

We have presented a novel time-domain description of passive mode-locking in electrically-driven verticalexternal-cavity surface-emitting lasers (VECSELs). The partially-integrated description analytically treats the bidirectional traveling wave equations in all linear layers while the quantum wells reduces to a nonlinear boundary condition. In the time-domain, the model is reduced to a set of differential equations coupled to an algebraic map, physically representing the delayed injection from one vertical cavity into its counterpart. The effects of dispersion and nonlinear saturation imposed by the vertical cavities are naturally accounted for by directly describing the evolution of the intracavity electric fields. In this sense, the description fills the gap between numerical iterative models and more sophisticated finite-difference theories.

Our model enables a thorough understanding of the mode-locking onset and the role played by the different design parameters on the mode-locking performance: pulsewidth, time-bandwidth product, phase noise, etc. Stable mode-locked pulses of $\sim 10 \mathrm{ps}$ temporal duration are obtained at $15 \mathrm{GHz}$ repetition rate. These results are in good agreement with reported experiments. The linear stability analysis of the compound-cavity modes correctly provides the first onset of instability characterized by multimode emission. Fully-developed modelocked pulses appear beyond this point. We find that mode-locking starts near the laser threshold when the saturation energies in the emitter and absorber are adjusted through their saturation fluences, beam spot sizes, and standing wave intensities in the cavities.

Finally, we would like to mention other potential applications and extensions of the model. Transverse effects in the present model have been taken into account at a level that ensures accuracy and efficiency. The range of single transverse mode operation could be determined by allowing the oscillation of several transverse modes in the structure. For Gaussian mode operation, the influence of inhomogeneous gain saturation on the pulsewidth and pulse stability could be investigated by including the spatial hole-burning effects. Temperature effects shifts the main resonance towards the blue side of the gain spectrum, which causes thermal roll-over and provides different resonant wavelengths in both cavities. Therefore, the structure must provide good current confinement around the symmetry axis in order to reduce the effective active area and, in turn, the laser threshold current. Our 
approach can be also generalized in order to investigate the conditions required for high-frequency mode-locking in monolithically integrated devices and to export the concepts to the $1550 \mathrm{~nm}$ wavelength.

\section{APPENDIX A. ITERATION FUNCTIONS FOR THE INJECTION FIELDS}

The functions $a_{1,2}(\omega)$ and $p_{1,2}(\omega)$ define the iteration of the injection fields $Y_{A, P}$

$$
\begin{aligned}
& a_{1}(\omega)=\left(r_{p}^{\prime}-t_{p} t_{p}^{\prime} \frac{e^{i 2 k L_{w p}}}{1+\tilde{r}_{p}}\right) \frac{t_{a}^{\prime} e^{i q L_{w a}}\left(1+\tilde{r}_{1}\right)}{t_{p}^{\prime} e^{i k L_{w p}}\left(1+\tilde{r}_{2}\right)}, \quad a_{2}(\omega)=t_{p} t_{a}^{\prime} e^{i\left(q L_{w a}+k L_{w p}\right)} \frac{\left(1+\tilde{r}_{1}\right)}{\left(1+\tilde{r}_{p}\right)}, \\
& p_{1}(\omega)=\left(r_{a}^{\prime}-t_{a} t_{a}^{\prime} \frac{e^{i 2 q L_{w a}}}{1+\tilde{r}_{a}}\right) \frac{t_{p}^{\prime} e^{i k L_{w p}}\left(1+\tilde{r}_{2}\right)}{t_{a}^{\prime} e^{i q L_{w a}}\left(1+\tilde{r}_{1}\right)}, \quad p_{2}(\omega)=t_{a} t_{p}^{\prime} e^{i\left(q L_{w a}+k L_{w p}\right)} \frac{\left(1+\tilde{r}_{2}\right)}{\left(1+\tilde{r}_{a}\right)} .
\end{aligned}
$$

The following notation is used: primed symbols stand for external reflectivity and transmissivity, $\tilde{r}_{1}=r_{1} \exp \left(i 2 q\left(L_{a}-\right.\right.$ $\left.\left.L_{w a}\right)\right), \tilde{r}_{a}=r_{a} \exp \left(i 2 q L_{w a}\right), \tilde{r}_{2}=r_{2} \exp \left(i 2 k\left(L_{p}-L_{w p}\right)\right)$, and $\tilde{r}_{p}=r_{p} \exp \left(i 2 k L_{w p}\right), q=\frac{\omega}{c} n_{a}$ and $k=\frac{\omega}{c} n_{p}$.

\section{APPENDIX B. $\tilde{N}_{I J}$ MATRIX}

The coefficients of the matrix $\tilde{N}_{i j}(\Omega)$ are functions of the SVE frequency $\Omega=\omega-\omega_{0}$ that read

$$
\begin{aligned}
& \tilde{N}_{11}(\Omega)=1-r_{1} r_{a} e^{i 2 q_{0} L}-\frac{\xi^{2} e^{i 2 \Omega \tau_{e}}}{\Delta(\Omega)} a_{1}\left(\omega_{0}\right) p_{2}\left(\omega_{0}\right), \quad \tilde{N}_{12}(\Omega)=\frac{\xi e^{i \Omega \tau_{e}}}{\Delta(\Omega)} a_{2}\left(\omega_{0}\right) \\
& \tilde{N}_{21}(\Omega)=\frac{\xi e^{i \Omega \tau_{e}}}{\Delta(\Omega)} p_{2}\left(\omega_{0}\right), \quad \tilde{N}_{22}(\Omega)=1-r_{2} r_{p} e^{i 2 k_{0} L_{p}}-\frac{\xi^{2} e^{i 2 \Omega \tau_{e}}}{\Delta(\Omega)} a_{2}\left(\omega_{0}\right) p_{1}\left(\omega_{0}\right)
\end{aligned}
$$

with $\Delta(\Omega)=1-\xi^{2} e^{i 2 \Omega \tau_{e}} a_{1}\left(\omega_{0}\right) p_{1}\left(\omega_{0}\right)$ and $\xi=\zeta t_{b s} \exp \left(i \omega_{0} \tau_{e}\right)$.

\section{ACKNOWLEDGMENTS}

The work by J.M. is supported by the Consejo Superior de Investigaciones Científicas through the program I3P-PC2003 and the project CONOCE2: FIS2004-00953. S.B. acknowledges support from the project TIC200204255-C04-01 and COST288 action "nanoscale and ultrafast photonics". The authors acknowledge fruitful discussions with K. Yvind, T. Greibe, J. Mørk, J. Javaloyes and E. Avrutin.

\section{REFERENCES}

1. R. Häring, R. Paschotta, A. Aschwanden, E. Gini, F. Morier-Genoud, and U. Keller, "High-power passively mode-locked semiconductor lasers," IEEE J. Quantum Electron. 38, pp. 1268-1275, 2002.

2. A. Garnache, S. Hoogland, A. C. Tropper, I. Sagnes, G. Saint-Girons, and J. S. Roberts, "Sub-500-fs solitonlike pulse in a passively mode-locked broadband surface-emitting laser with $100 \mathrm{~mW}$ average power," Appl. Phys. Lett. 80, pp. 3892-3894, 2002.

3. C. Symonds, J. Dion, I. Sagnes, M. Dainese, M. Strassner, L. Loreu, and J.-L. Oudar, "High performance $1.55 \mu \mathrm{m}$ vertical external cavity surface emitting laser with broadband integrated dielectric-metal mirror," Electron. Lett. 40, pp. 734-735, 2004.

4. K. Jasim, Q. Zhang, A. V. Nurmikko, A. Mooradian, G. Carey, W. Ha, and E. Ippen, "Passively modelocked vertical extended cavity surface emitting diode laser," Electron. Lett. 39, pp. 373-374, 2003.

5. K. Jasim, Q. Zhang, A. V. Nurmikko, E. Ippen, A. Mooradian, G. Carey, and W. Ha, "Picosecond pulse generation from passively modelocked vertical cavity diode laser at up to $15 \mathrm{GHz}$ repetition rate," Electron. Lett. 40, pp. 34-36, 2004.

6. A. C. Tropper, H. D. Foreman, A. Garnache, K. G. Wilcox, and S. H. Hoogland, "Vertical-external-cavity semiconductor lasers," J. Phys. D: Appl. Phys. 37, pp. R75-R85, 2004.

7. U. Keller, K. J. Weingarten, F. X. Kärtner, D. Kopf, B. Braun, I. D. Jung, R. Fluck, C. Hönninger, N. Matuschek, and J. Aus der Au, "Semiconductor saturable absorber mirrors (SESAM's) for femtosecond to nanosecond pulse generation in solid-state lasers," IEEE J. Selected Topics Quantum Electron. 2, pp. 435453, 1996. 
8. S. Hoogland, S. Dhanjal, A. C. Tropper, J. S. Roberts, R. Häring, R. Paschotta, F. Morier-Genoud, and U. Keller, "Passively mode-locked diode-pumped surface-emitting semiconductor lasers," IEEE Photon. Technol. Lett. 12, pp. 1135-1137, 2000.

9. R. Häring, R. Paschotta, E. Gini, F. Morier-Genoud, D. Martin, H. Melchior, and U. Keller, "Picosecond surface-emitting semiconductor laser with $>200 \mathrm{~mW}$ average output power," Electron. Lett. 37, pp. 766$768,2001$.

10. R. Paschotta, R. Häring, A. Garnache, S. Hoogland, A. C. Tropper, and U. Keller, "Soliton-like pulseshaping mechanism in passively mode-locked surface-emitting semiconductor lasers," Appl. Phys. B 75, pp. 445-451, 2002.

11. D. Lorenser, H. J. Unold, D. J. H. C. Maas, A. Aschwanden, R. Grange, R. Paschotta, D. Ebling, E. Gini, and U. Keller, "Towards wafer-scale integration of high repetition rate passively mode-locked surface-emitting semiconductor lasers," Appl. Phys. B 79, pp. 927-932, 2004.

12. Q. Zhang, K. Jasim, A. V. Nurmikko, A. Mooradian, G. Carey, W. Ha, and E. Ippen, "Operation of a passively mode-locked extended-cavity surface-emitting diode laser in multi-Ghz regime," IEEE Photon. Technol. Lett. 16, pp. 885-887, 2004.

13. Q. Zhang, K. Jasim, A. V. Nurmikko, E. Ippen, A. Mooradian, G. Carey, and W. Ha, "Characteristics of a high-speed passively mode-locked surface-emitting semiconductor InGaAs laser diode," IEEE Photon. Technol. Lett. 17, p. 525, 2005.

14. C. J. Chen, P. K. A. Wai, and C. R. Menyuk, "Self-starting of passively mode-locked lasers with fast saturable absorbers," Opt. Lett. 20, pp. 350-352, 1995.

15. M. Bahl, H. Rao, N. C. Panoiu, and J. R. M. Osgood, "Simulation of mode-locked surface-emitting lasers through a finite-difference time-domain algorithm," Opt. Lett. 29, pp. 1689-1691, 2004.

16. M. Bahl, N. C. Panoiu, and J. R. M. Osgood, "Modeling ultrashort field dynamics in surface-emitting lasers by using finite-difference time-domain method," IEEE J. Quantum Electron. 41(10), pp. 1244-1252, 2005.

17. J. Mulet and J. Mørk, "Analysis of timing jitter in external-cavity mode-locked semiconductor lasers," IEEE J. Quantum Electron. 42(3), pp. 249-256, 2006.

18. C. Campanario and E. Avrutin, "An efficient model for dynamic simulation of mode-locked vertical external cavity surface emitting lasers," pp. 85-86, Numerical Simulation of Optoelectronic Devices, on 19-22 Sept., 2005.

19. H. A. Haus, "Theory of mode locking with a fast saturable absorber," J. Appl. Phys. 46, pp. 3049-3058, 1975.

20. J. Mulet and S. Balle, "Mode locking dynamics in electrically-driven vertical-external-cavity surface-emitting lasers," IEEE J. Quantum Electron. 41(9), pp. 1148-1156, 2005.

21. A. E. Siegman, Lasers, University science books, Mill Valley, CA, 1986.

22. T. E. Sale, "Cavity and reflector design for vertical cavity surface emitting lasers," IEE Proc.Optoelectron. 142, pp. 37-43, 1995.

23. J. F. Martins-Filho, E. A. Avrutin, C. N. Ironside, and J. S. Roberts, "Monolithic multiple colliding pulse mode-locked quantum-well lasers: Experiment and theory," IEEE J. Selected Topics Quantum Electron. 1, pp. 539-551, 1995.

24. E. A. Avrutin, J. H. Marsh, and J. M. Arnold, "Modelling of semiconductor laser structures for passive harmonic mode locking at terahertz frequencies," Int. J. Optoelectron. 10, pp. 427-432, 1995.

25. S. Balle, "Analytical description of spectral hole-burning effects in active semiconductors," Opt. Lett. 21, pp. 1923-1925, 2002.

26. J. Hudgings, R. J. Stone, S. F. Lim, G. S. Li, W. Yuen, K. Y. Lau, and C. J. Chang-Hasnain, "The physics of negative differential resistance of an intracavity voltage-controlled absorber in a vertical-cavity surface-emitting laser," Appl. Phys. Lett. 73, pp. 1796-1798, 1998.

27. J. Mulet and S. Balle, "Spatiotemporal optical model of VCSELs the in presence of polarization effects," IEEE J. Quantum Electron. 38, pp. 291-305, 2002.

28. S. F. Yu, Analysis and design of vertical cavity surface emitting lasers, John Wiley and Sons, Hoboken, NY, 2003.

29. J. Mulet, C. Masoller, and C. R. Mirasso, "Modeling bidirectionally coupled single-mode semiconductor lasers," Phys. Rev. A 65, p. 063815, 2002. 